\title{
Shifting Paradigms in Minimally Invasive Surgery: Applications of Transanal Natural Orifice Transluminal Endoscopic Surgery in Colorectal Surgery
}

\author{
Grace Clara Lee, MD ${ }^{1}$ Patricia Sylla, MD ${ }^{1}$ \\ ${ }^{1}$ Division of Gastrointestinal Surgery, Department of Surgery, \\ Massachusetts General Hospital, Boston, Massachusetts \\ Clin Colon Rectal Surg 2015;28:181-193.
}

\begin{abstract}
Address for correspondence Patricia Sylla, MD, FACS, FASCRS, Division of Colon and Rectal Surgery, Department of Surgery, Mount Sinai Hospital, 5 East 98th Street, Box 1259, New York, NY 10029 (e-mail: patricia.sylla@mountsinai.org).
\end{abstract}

\begin{abstract}
Since the advent of laparoscopy, minimally invasive techniques such as single port laparoscopy, robotics, endoscopically assisted laparoscopy, and transanal endoscopic surgery continue to revolutionize the field of colorectal surgery. Transanal natural orifice transluminal endoscopic surgery (NOTES) represents a further paradigm shift by combining the advantages of these earlier techniques to reduce the size and number of abdominal incisions and potentially optimize rectal dissection, especially with respect to performance of an oncologically adequate total mesorectal excision (TME) for rectal cancer. Since the first experimental report of transanal rectosigmoid resection in 2007 , the potential impact of transanal NOTES in colorectal surgery has been extensively investigated in experimental models and recently transitioned to clinical application. There have been 14 clinical trials of transanal TME (taTME) for rectal cancer that have demonstrated the feasibility and preliminary oncologic safety of this approach in carefully selected patients, with results comparable to outcomes after laparoscopic and open TME, including cumulative intraoperative and postoperative complication rates of 5.5 and $35.5 \%$, respectively, $97.3 \%$ rate of complete or near-complete specimens, and $93.6 \%$ rate of negative margins. Transanal NOTES has also been safely applied to proctectomy and colectomy for benign indications. The consensus among published

Keywords

- transanal

- NOTES

- TME

- mesorectal excision

- proctectomy series suggests that taTME is most safely performed with transabdominal assistance by surgeons experienced with laparoscopic TME, transanal endoscopic surgery, and sphincter-preserving techniques including intersphincteric resection. Future applications of transanal NOTES may include evolution to a pure endoscopic transanal approach for TME, colectomy, and sentinel lymph node biopsy for rectal cancer, with a potential role for robotic assistance.
\end{abstract}

Though adoption of the surgical principles of total mesorectal excision (TME) in combination with chemoradiation for locally advanced disease has continued to improve local control and survival rates of resectable rectal cancer, radical resection continues to be associated with high morbidity and mortality. ${ }^{1,2}$ Standard multiport and robotic-assisted laparoscopy, although oncologically safe and associated with shorter length of hospital stay (LOS) than open resections, have not had a measurable impact on the incidence of postoperative complications, sexual and urinary dysfunction,
Issue Theme Evolving and Emerging Technologies in Colon and Rectal Surgery; Guest Editors: Thomas E. Cataldo, MD, FACS, FASCRS; Deborah Nagle, MD, FACS, FASCRS
Copyright $\odot 2015$ by Thieme Medical Publishers, Inc., 333 Seventh Avenue, New York, NY 10001, USA. Tel: +1(212) 584-4662.
DOI http://dx.doi.org/ 10.1055/s-0035-1555009. ISSN 1531-0043. 
or quality of life. In addition, widespread adoption of laparoscopic techniques in colorectal surgery has been severely limited by the technical complexity and steep learning curve required to gain expertise in minimally invasive pelvic resections. In an effort to harness the advantages of a minimally invasive approach to benefit patients with colorectal pathology, transanal natural orifice transluminal endoscopic surgery (NOTES) has been explored, with promising preliminary results, particularly when used for rectal cancer. Here, we review the history of NOTES, evolution of minimally invasive and transanal approaches applied to colorectal diseases, results of transanal NOTES proctectomy clinical trials for both malignant and benign indications, current indications for NOTES proctectomy, and future applications of NOTES.

\section{History of Natural Orifice Transluminal Endoscopic Surgery}

The first clinical report of transgastric appendectomy performed using a flexible endoscope by Reddy and Rao in 2004 ignited worldwide interest in NOTES as a method of extending flexible endoscopy into the realm of minimally invasive intra-abdominal surgery. ${ }^{3}$ Over the subsequent few years, NOTES was intensely explored in animal models with the hope of significantly reducing access trauma by operating through a natural orifice instead of the abdominal or thoracic wall. ${ }^{4}$ Theoretical advantages of this approach included decreased postoperative pain, faster postoperative recovery, decreased postoperative complications, including wound infections and incisional hernias, as well as improved cosmesis., 3

After experimental evidence in animal models demonstrated the feasibility and safety of peritoneal access via transoral, transanal, transurethral, and transvaginal routes, NOTES cautiously entered clinical practice. ${ }^{4,6,7}$ While transgastric approaches were initially most popular, the risks of creating a gastrotomy solely for access purposes were deemed prohibitively high. ${ }^{3}$ Transvaginal NOTES procedures quickly surpassed all other transluminal access routes with thousands of transvaginal cholecystectomies performed to date using either rigid or flexible instrumentation with excellent safety profile and patient satisfaction scores. ${ }^{3,8-10}$ Transanal and transcolonic NOTES were adopted more slowly because of initial concerns about risk of infection and reliable colorectal incisional closure. Extensive evidence in swine survival models, however, demonstrated the safety of transcolonic NOTES peritoneoscopy and other intra-abdominal procedures, as long as adequate closure of the colotomy was achieved..$^{11-15}$

Colorectal resection ensued as the most logical application of transanal NOTES. Beyond the theoretical benefits common to all NOTES procedures, transanal NOTES had the distinct advantage of creating the viscerotomy within the diseased target organ and incorporating it into the colorectal anastomosis. Several endoscopic prototypes designed to achieve safe transluminal access, dissection, and endoscopic closure were explored without successful transition to clinical trial, highlighting the obstacles faced by NOTES with performing complex endoscopic procedures with maladapted instrumentation. ${ }^{16-18}$ In this setting, the report by Whiteford et al in 2007, describing rectosigmoid resection in three human cadavers using a purely transanal approach with transanal endoscopic microsurgery (TEM), was a turning point in the evolution of transanal NOTES. ${ }^{19}$ It highlighted the potential of performing complex colorectal dissection using existing transanal endoscopic platforms, thus enabling experimental and then clinical trials using this innovative approach.

\section{Evolution of Minimally Invasive Approaches to Rectal Surgery}

The development of transanal NOTES approach for rectal resection comes in the context of efforts to minimize the morbidity and mortality of open rectal surgery. Three decades following its original description by Heald, ${ }^{20} \mathrm{TME}$ has become the gold standard for curative rectal cancer resection. However, it continues to be associated with a 1 to $6 \%$ mortality rate as well as high perioperative morbidity, including anastomotic leak (0-21\%), wound complications $(0-47 \%)$, and hernia formation $(0-11 \%)$, as well as functional disorders, including defecatory $(0.5-37 \%)$, urinary (5-14\%), and sexual dysfunction (33-36\%). ${ }^{1,2,21-34}$

Relative to open TME, laparoscopic TME is associated with decreased postoperative pain, decreased LOS, and faster return of bowel function, with similar oncologic outcomes based on large randomized controlled trials, including the CLASSIC and COLOR II trials. ${ }^{35-37}$ However, laparoscopic TME has not been widely adopted because of the difficulty of the pelvic dissection, long operative time, steep learning curve, and minimal impact of the laparoscopic approach on functional outcomes. ${ }^{1,38-42}$ Regardless of the specific reasons for the lack of wider adoption, it is estimated that well under $30 \%$ of the rectal cancer resections in the United States are performed using laparoscopy, without significant improvement over the past decade, and persistently high conversion rates (15$30 \%)^{35-37}$ Single-port laparoscopy for colon resections has not been shown to provide advantages over traditional laparoscopy and has been minimally investigated for rectal resections because of technical difficulty. ${ }^{4-46}$ Preliminary data from large series on robotic TME suggest equivalent perioperative and oncologic outcomes relative to laparoscopic TME, but with decreased conversion rates to open surgery. ${ }^{47,48}$ However, robotic surgery is associated with significantly longer operative times and higher procedural costs, potentially problematic in this environment of health-care cost reduction. ${ }^{49}$ Furthermore, laparoscopic and robotic TME still require a sizeable abdominal incision for specimen removal, minimizing their wound-related benefits over open TME.

In addition to NOTES, natural orifice specimen extraction (NOSE) has gained increasing popularity, with many groups reporting successful transvaginal and transanal removal of colorectal resection specimens. ${ }^{50-57}$ Many of these procedures have been performed for benign indications, such as rectal prolapse and diverticulitis; ${ }^{58,59}$ however, there have also been several studies employing this technique for colorectal cancer resections. Park et al compared laparoscopic right colectomy with transvaginal versus transabdominal extraction $(n=68)$ and found decreased postoperative pain and LOS associated 
with transvaginal removal, though wound infections and intraabdominal abscesses were not significantly decreased. ${ }^{60}$ Leung et al $(n=70)$ conducted a randomized trial of laparoscopic left colectomy with transanal versus transabdominal extraction, finding decreased postoperative pain $(p=0.017)$ and wound infections $(p=0.005)$ with transanal NOSE. ${ }^{61}$ A large retrospective comparison $(n=432)$ by Liang et al of laparoscopic TME for rectal cancer with transanal or transabdominal specimen extraction found no difference in intraoperative complications ( $p=0.69$ ), postoperative complications ( $p=0.59$ ), LOS $(p=0.83)$, or 2-year local recurrence rates $(p=0.15) .{ }^{62,63}$ Thus, preliminary results show NOSE to be feasible for colorectal cancer resection, with possible advantages including reduction in postoperative pain, LOS, and wound infection rates.

\section{Evolution of Transanal Approaches to Rectal Surgery}

Alongside these minimally invasive transabdominal approaches to colorectal surgery, transanal techniques for the removal of rectal lesions have been developed. Over the last three decades, TEM has provided excellent endoscopic local control for proximal rectal lesions through improved visualization and full thickness resection, resulting in decreased fragmentation of specimens relative to conventional transanal excision. ${ }^{64-66}$ In addition, TEM has been associated with exceedingly low mortality and morbidity relative to radical proctectomy. ${ }^{67,68}$ More recently, transanal minimally invasive surgery (TAMIS), a newer technique that utilizes flexible and disposable transanal endoscopic platforms and accommodates traditional laparoscopic instruments, has accelerated the popularity of transanal endoscopic surgery worldwide. $^{69-72}$ Inherent limitations of TEM and TAMIS apply to all local excision techniques, namely the lack of mesorectal clearance with prohibitively high recurrence rates when used to resect T2 and more advanced rectal tumors. As a result, TEM and TAMIS are only indicated for resection of benign rectal lesions and carefully selected $\mathrm{T} 1$ rectal tumors with low-risk features, as local recurrence rates for those lesions after TEM are similar to rates after TME. ${ }^{73-81}$

Transanal NOTES expands the conventional indications of TEM and TAMIS by taking advantage of the exposure and access provided by transanal endoscopic platforms. Transanal rectal distention with $\mathrm{CO}_{2}$ combined with magnified TEM and TAMIS optics permits excellent visualization of tissue planes and precise tissue manipulation. ${ }^{82}$ Full thickness rectal dissection can be extended beyond the rectal wall to encompass the mesorectum, and pneumodissection facilitates identification of the presacral space and rectovaginal or rectoprostatic plane (-Fig. 1). This transanal endoscopic "down-to-up" approach may be particularly helpful in patients with a narrow pelvis and significant visceral obesity, in whom laparoscopic pelvic dissection would be challenging and where the risk for conversion to open surgery is the highest. ${ }^{43,83,84}$

With regard to low rectal cancers abutting the anorectal ring, transanal NOTES access is also expanding the application of intersphincteric resection (ISR). ISR has been used since the early 1990s as a sphincter-preserving approach for very low rectal tumors, and involves extending the rectal dissection into the intersphincteric plane and removing part or all the internal sphincter to achieve negative distal margins. ${ }^{85}$ ISR is combined with open, laparoscopic, or robotic transabdominal TME (TATA or transanal abdominotransanal resection) with equivalent oncologic outcomes as abdominoperineal resection (APR) for tumors lower than $4 \mathrm{~cm}$ from the anal verge (AV). ${ }^{86,87}$ Transanal NOTES is particularly well suited for intersphincteric completion proctectomy for inflammatory bowel disease (IBD) and for low rectal tumors when ISR would otherwise be indicated to achieve negative distal margins. Following intersphincteric dissection of the internal anal sphincter, the rectal stump is sutured and a transanal platform is inserted to complete the transanal NOTES proctectomy or TME. ${ }^{84,88}$ Unlike TATA, where more proximal mesorectal dissection is limited by poor exposure with conventional anal retractors, the transanal NOTES approach allows transanal completion of the rectal and mesorectal excision all the way toward the sacral promontory where the peritoneal reflection is opened and the abdominal cavity entered. Finally, size of rectum and width of pelvic inlet permitting transanal specimen extraction can be combined with transanal NOTES resection followed by transanal handsewn or stapled anastomosis, without the absolute need for an abdominal extraction site (-Fig. 1).

Thus, transanal NOTES combines the advantages of transanal endoscopic surgery (TEM and TAMIS), sphincter-preserving techniques including ISR, and NOSE. By facilitating identification of the distal resection margin and improving visualization, exposure and dissection of the perirectal and mesorectal planes, transanal NOTES may optimize the quality of rectal resections while minimizing the morbidity of these procedures.

\section{Clinical Trials of Transanal Natural Orifice Transluminal Endoscopic Surgery}

Numerous porcine survival and human cadaver trials have confirmed the safety and feasibility of a transanal NOTES approach to colorectal resection. ${ }^{19,89-98}$ In the largest cadaver study ( $n=32$ ), adequate TME was achieved in every cadaver and, when comparing different transanal approaches, the laparoscopic-assisted technique was found to be particularly important in minimizing organ injury and maximizing specimen length, ${ }^{97}$ leading to the subsequent use of laparoscopic assistance in many clinical trials.

Currently, transanal NOTES has been most thoroughly investigated for TME for rectal cancer. However, several reports have described both pure and laparoscopic-assisted transanal NOTES approaches to perform colorectal resections for benign indications.

\section{Transanal Natural Orifice Transluminal Endoscopic Surgery Proctectomy and Colectomy for Benign Disease}

Lacy et al were the first to report a successful laparoscopicassisted transanal NOTES total colectomy in a 36-year-old 

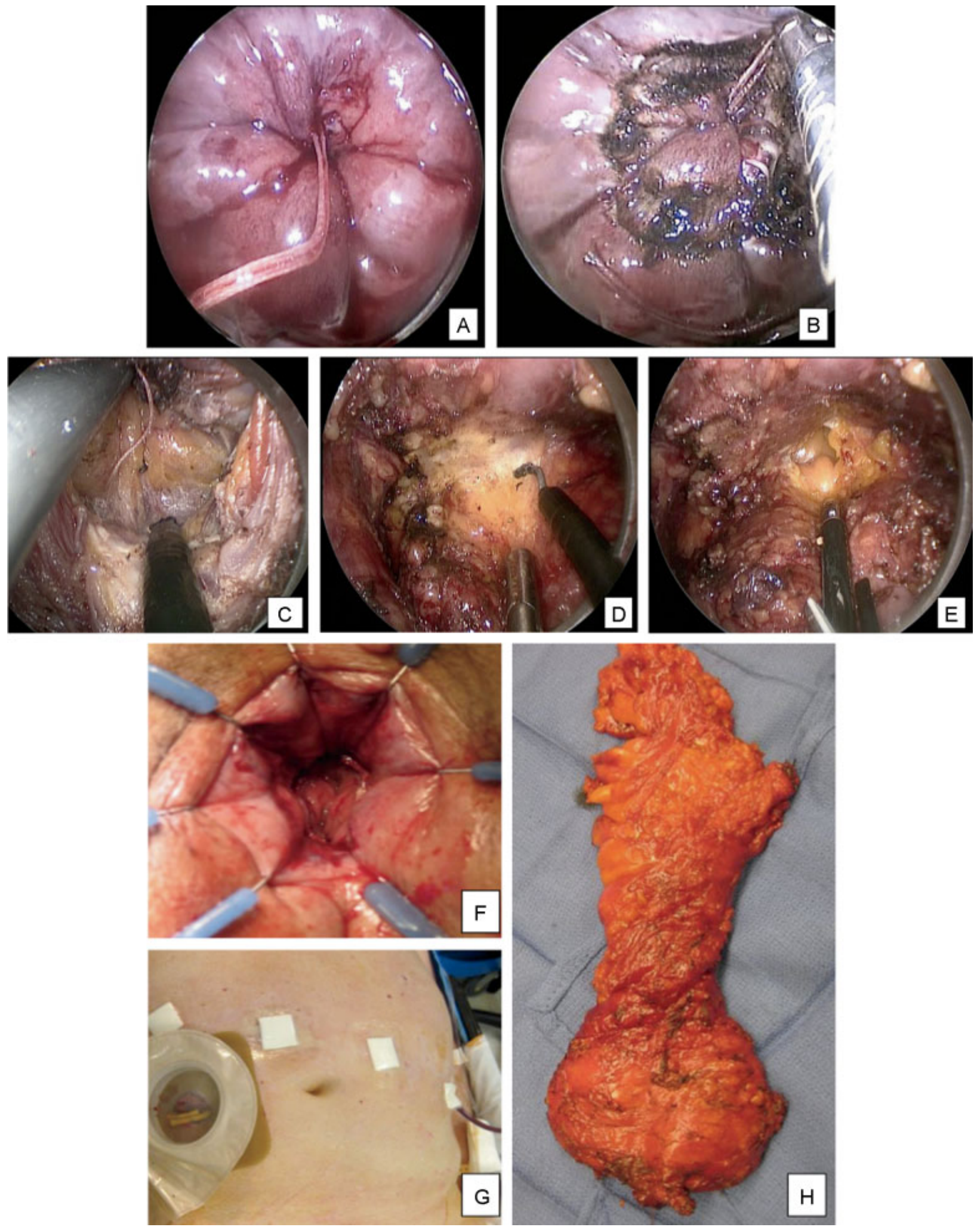

Fig. 1 Laparoscopic-assisted taTME in an obese male patient with a T2N0 low rectal cancer. The rectum is sutured closed below the tumor with a purse string located just above the dentate line $(\mathrm{A})$ and the transanal endoscopic platform is inserted. After $\mathrm{CO}_{2}$ insufflation of the rectum, the rectal mucosa is scored circumferentially with monopolar cautery (B). Full-thickness rectal and mesorectal dissection is completed posteriorly along the presacral space (C), laterally, and anteriorly along the rectoprostatic plane (D) until the peritoneal reflection is reached and the abdominal cavity entered anteriorly (E). Rectal and mesorectal dissection is completed using a combined abdominal and transanal approach, and the specimen is transected transanally if feasible. Stapled coloanal anastomosis is performed (F) followed by a protective diverting loop ileostomy (G). A complete TME is achieved with negative margins and 25 negative lymph nodes (H). taTME, transanal total mesorectal excision; TME, total mesorectal excision. 
man with medically refractory ulcerative colitis. ${ }^{99}$ Total operative time was 240 minutes and there were no surgical complications.

Fuchs et al performed laparoscopic-assisted transanal colon resections in 15 patients with additional rectopexy in 11 of the 15 patients for benign indications (full-thickness rectal prolapse, internal rectal intussusception with pelvic obstruction, recurrent sigmoid diverticulitis, and severe slow-transit constipation). ${ }^{59}$ There were no intraoperative complications except for one conversion to full laparoscopy with a minilaparotomy to remove the bulky specimen in a patient with diverticulitis. Mean operative time was 131 minutes. There were two postoperative complications (postoperative ileus that resolved with conservative management and intra-abdominal bleeding that required a transfusion but no other intervention). At 6-month follow-up, the median Gastrointestinal Quality of Life Index increased significantly $(p<0.05)$ and no patient reported any functional complaints.

Liyanage et al report a series of 12 patients who underwent transanal completion proctectomy for inflammatory bowel disease, rectal adenomas, or radiation proctitis. ${ }^{88}$ All procedures were performed purely transanally except in two patients. Mean rectal stump length was $17.8 \mathrm{~cm}$. Median LOS was 5.5 days. There were seven postoperative complications (five delayed wound healing, one temporary incarceration of a parastomal hernia, and one colocutaneous fistula to the perineum in the patient with radiation proctitis, which required operative intervention).

Wolthuis et al describe a series of 14 transanal rectal excisions, laparoscopically assisted in 11 of the cases, for benign and malignant indications. ${ }^{100}$ Among the nine patients with benign disease (inflammatory bowel disease, fistula, adenoma, fecal incontinence, and anastomotic complications), ISR with coloanal anastomosis was performed in six patients and proctectomy with end colostomy was performed in the remaining three patients. Mean operating time was 146 minutes. Two patients required conversion to open surgery because of extensive adhesions. Mean LOS was 7.6 days and minor complications occurred in 4 of the 14 patients.

Thus, these early trials suggest that transanal NOTES is feasible for proctectomy and colectomy in carefully selected patients with benign disease, and is most commonly and safely performed with laparoscopic assistance.

\section{Transanal Natural Orifice Transluminal Endoscopic Surgery Total Mesorectal Excision for Rectal Cancer} With regard to rectal cancer resection, there have been an increasing number of transanal NOTES TME (taTME) cases performed since the first clinical report of laparoscopicassisted taTME in a 76-year-old woman with rectal cancer. ${ }^{101}$ Thus far, 14 clinical series have been reported from groups in the United States, Asia, and Europe, involving a total of 110 patients (-Table 1)..$^{34,43,82,84,101-112}$ Sixty-seven percent of the patients were male, mean patient age was 61.5 years, mean body mass index (BMI) was $25.8 \mathrm{~kg} / \mathrm{m}^{2}$, and $80.6 \%$ of the patients received neoadjuvant therapy.
In general, most series selected patients based on tumorspecific criteria including low- and mid-rectal resectable tumors (staged by pelvic magnetic resonance imaging [MRI] and/or endorectal ultrasound), with no evidence of metastases on computed tomographic (CT) scan, and excluded patients with $\mathrm{BMI}>35 \mathrm{~kg} / \mathrm{m}^{2}$ and previous extensive abdominal or pelvic surgery. By contrast, two series intentionally selected patients with anatomical features predictive of difficult rectal dissection (narrow pelvis, BMI $>30 \mathrm{~kg} / \mathrm{m}^{2}$, and large prostate). ${ }^{43,108}$ Rouanet et al also selected patients with T4 and recurrent tumors, including 25 patients (75\%) with predicted circumferential resection margins $\leq 1 \mathrm{~mm}$ based on MRI and 3 patients (10\%) with known metastases before surgery. ${ }^{108}$ Overall, in all 14 series, $44.1 \%$ of the tumors were located in the lower rectum ( $<5 \mathrm{~cm}$ from the AV), 52.7\% were in the middle ( $5-10 \mathrm{~cm}$ from the $\mathrm{AV})$, and $3.2 \%$ were high ( $>10 \mathrm{~cm}$ from the AV). Of the 43 patients for whom preoperative nodal status was reported, $41.9 \%$ had positive nodes. On final pathology, $37.7 \%$ of the patients had positive nodes.

Mean operative time was 265.6 minutes and mean estimated blood loss was $138.2 \mathrm{~mL}$. Most of the series utilized a hybrid NOTES technique; 89 cases were laparoscopic assisted, 6 were robotic assisted, 3 were open assisted, and 12 were unassisted, purely transanal NOTES. Despite the majority of these trials being transabdominally assisted, most of the rectal dissection was performed transanally, hence their classification as transanal NOTES, as opposed to NOSE. The rectum was occluded transanally with a purse-string suture before making the enterotomy in $92.7 \%$ of the patients. In $69.1 \%$ of the patients, the splenic flexure was partially or fully mobilized. Seventy-seven percent of the patients received a diverting ileostomy. All groups extracted the specimen transanally, except Dumont et al who utilized the future ileostomy site and Atallah et al who used a Pfannenstiel incision. $43,83,102$

The overall intraoperative complication rate across all 14 clinical series of taTME was $5.5 \%$, with 2 conversions to open surgery, 2 urethral injuries, 1 possible air embolism, and 1 episode of pneumatosis of the small bowel mesentery (-Table 2). Rouanet et al, who published the series of highrisk patients, reported five of the six intraoperative events, explaining that the complications occurred early in the surgeons' learning curve and in patients with large, fixed tumors. ${ }^{108}$ The rate of conversion to open surgery was $1.8 \%$, which compares favorably to published conversion rates after laparoscopic TME of 0 to $34 \%$. 1,36-38,42,113,114

The cumulative postoperative complication rate across all series of taTME was 35.5\%, comparable to published morbidity rates of 21 to $44 \%$ and 24 to $51 \%$ after laparoscopic and open TME, respectively. ${ }^{1,36,37,42,113,115}$ Morbidity included 12 infectious (10.9\%) and 5 anastomotic (4.5\%) complications. Six patients experienced urinary dysfunction (5.5\%), likely because of pelvic nerve injury and comparable to reported rates after laparoscopic (up to 20\%) and open (5-14\%) TME. ${ }^{116,117}$ Mean LOS after taTME was 9.5 days, no patient died within 30 days of surgery, and no patient developed incisional hernias during follow-up. 


\begin{tabular}{|c|c|c|c|c|c|c|c|c|c|c|c|c|c|c|c|}
\hline 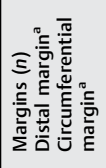 & 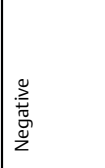 & 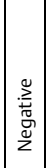 & 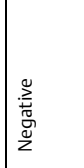 & 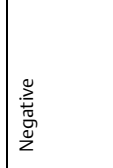 & 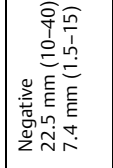 & 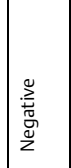 & 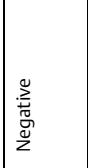 & 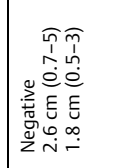 & 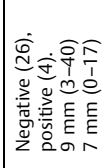 & 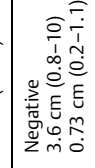 & $\frac{\alpha}{z}$ & 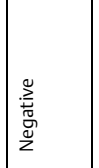 & 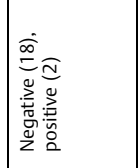 & 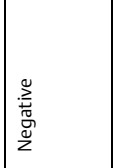 & 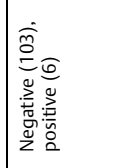 \\
\hline 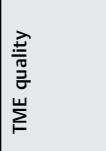 & 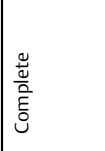 & $\mid$\begin{tabular}{|c|}
$\mid \frac{\mathrm{g}}{\mathrm{\omega}}$ \\
$\underline{\mathrm{E}}$ \\
$\mathrm{G}$ \\
\end{tabular} & 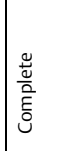 & 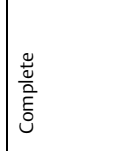 & 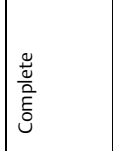 & 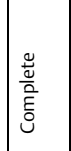 & 造 & 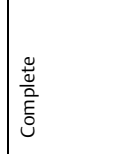 & 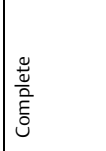 & 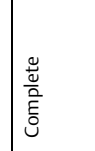 & 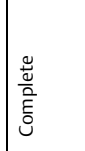 & 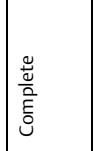 & 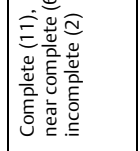 & 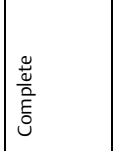 & 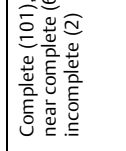 \\
\hline 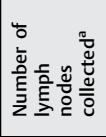 & $\approx$ & 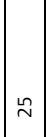 & $\stackrel{\varrho}{\circ}$ & $\stackrel{\simeq}{=}$ & $\begin{array}{l}\widehat{\widetilde{N}} \\
\infty \\
\infty \\
\underline{\infty}\end{array}$ & $\frac{\alpha}{z}$ & 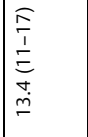 & 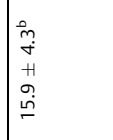 & $\begin{array}{l}\widehat{\widetilde{v}} \\
\tilde{\omega} \\
\stackrel{\infty}{m} \\
\underline{\underline{m}}\end{array}$ & 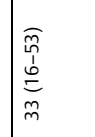 & $\varrho$ & $\simeq$ & 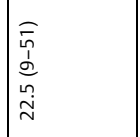 & 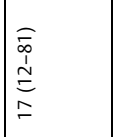 & 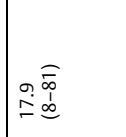 \\
\hline 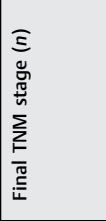 & 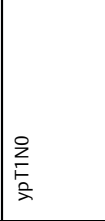 & $\frac{2}{2}$ & 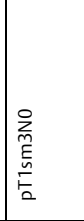 & $\begin{array}{l}\bar{a} \\
0 \\
\sum_{0} \\
\sum_{0} \\
\underline{a}\end{array}$ & $\ddot{z}$ & 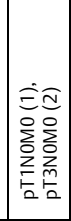 & 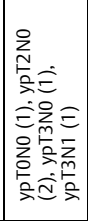 & 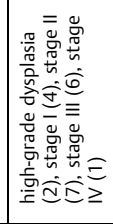 & 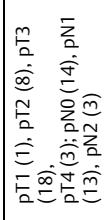 & 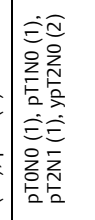 & 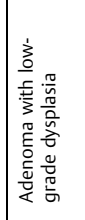 & 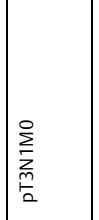 & 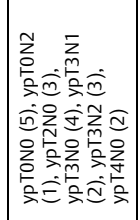 & 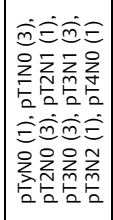 & 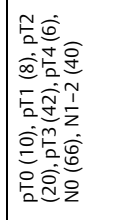 \\
\hline 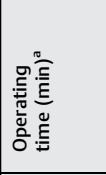 & $\stackrel{i}{i}$ & $\stackrel{D}{\sim}$ & 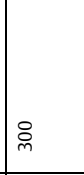 & 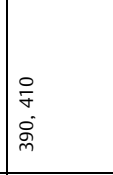 & 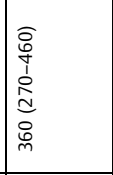 & 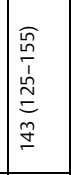 & 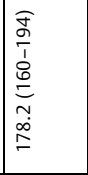 & 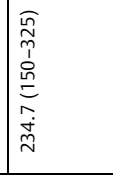 & 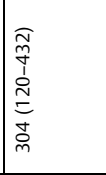 & 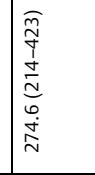 & $\stackrel{\circ}{\circ}$ & 省 & 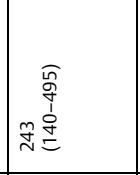 & 亭 & 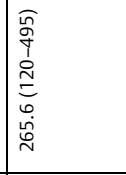 \\
\hline 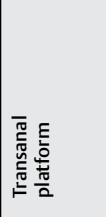 & 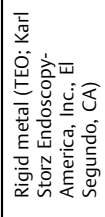 & 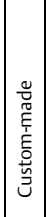 & 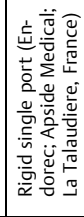 & 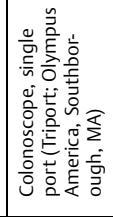 & 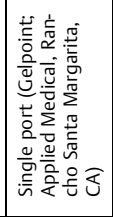 & 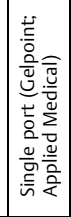 & 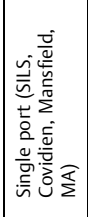 & 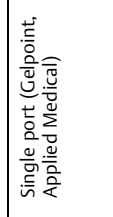 & 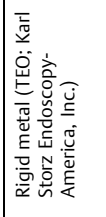 & 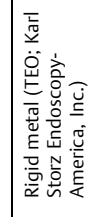 & 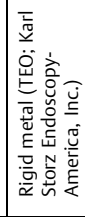 & 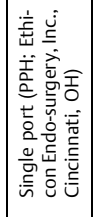 & 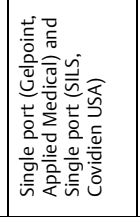 & 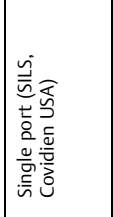 & 1 \\
\hline 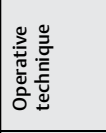 & $\leq$ & \pm & $\leq$ & $I$ & $I$ & $\leq$ & 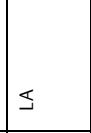 & $\leq$ & \pm & $s$ & a & $\therefore$ & 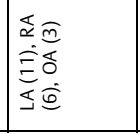 & 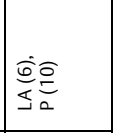 & 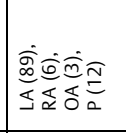 \\
\hline 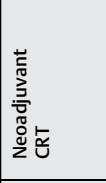 & $\stackrel{\check{\nu}}{\rightleftharpoons}$ & $\stackrel{\check{\Delta}}{\rightleftharpoons}$ & z & 2 & ֻั & 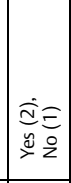 & ๖ั & 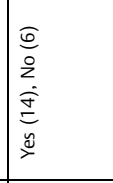 & 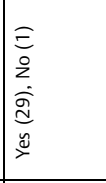 & 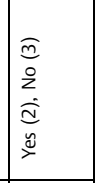 & 2 & $\frac{\alpha}{z}$ & 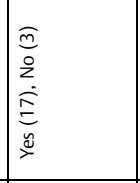 & $\frac{\alpha}{2}$ & 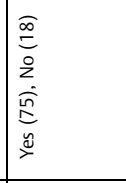 \\
\hline 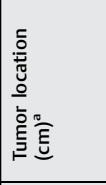 & 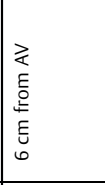 & 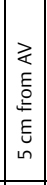 & 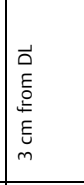 & 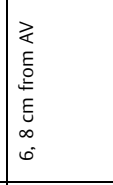 & 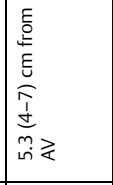 & 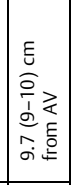 & 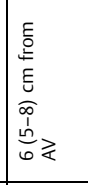 & 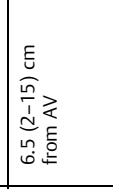 & 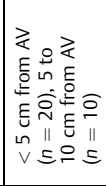 & 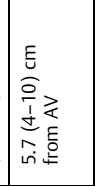 & 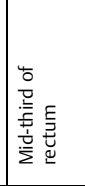 & $\begin{array}{l}\gtrless \\
\gtrless \\
E \\
\vdots \\
0 \\
E \\
\vdots \\
r\end{array}$ & 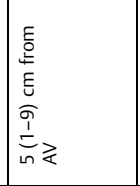 & 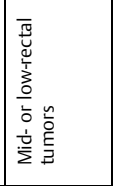 & 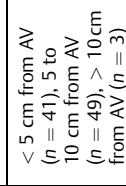 \\
\hline 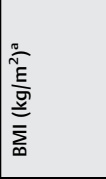 & i & $\approx$ & $\stackrel{\sim}{\sim}$ & $\frac{\alpha}{2}$ & 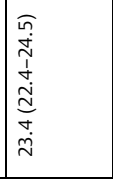 & 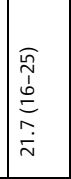 & $\frac{\alpha}{z}$ & 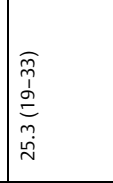 & 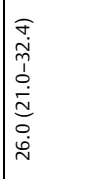 & 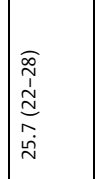 & $\frac{\alpha}{2}$ & i & 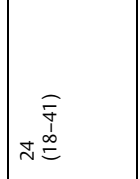 & 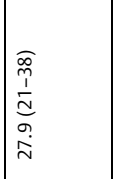 & 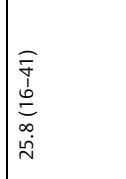 \\
\hline 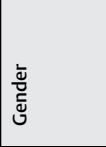 & $\sqcup$ & $\Sigma$ & $\sqcup$ & $\begin{array}{l}\dot{\bar{E}} \bar{\Sigma} \\
\dot{\Sigma} \bar{\psi}\end{array}$ & $\frac{\Phi}{\Sigma}$ & \begin{tabular}{|l}
$\dot{\bar{E}} \bar{\alpha}$ \\
$\bar{\Sigma}$
\end{tabular} & $\frac{\hat{\bar{m}}}{\bar{\Sigma} \widehat{\bar{y}}}$ & 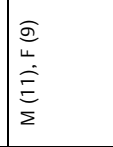 & $\begin{array}{l}\frac{a}{o} \\
\frac{m}{\Sigma}\end{array}$ & $\frac{\hat{\bar{m}} \overline{\mathrm{J}}}{\bar{z}}$ & 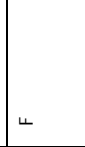 & 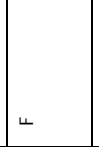 & 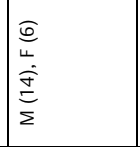 & 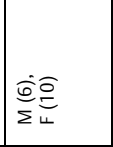 & 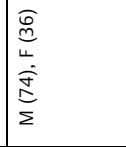 \\
\hline 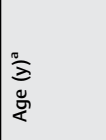 & 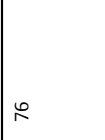 & f & タ & $\begin{array}{l}m \\
\text { m } \\
\dot{f} \\
w\end{array}$ & 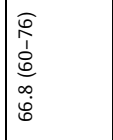 & 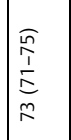 & $\mid \begin{array}{l}5 \\
5 \\
0 \\
0 \\
0 \\
0 \\
0 \\
0\end{array}$ & 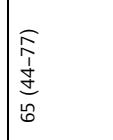 & 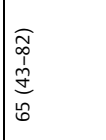 & \begin{tabular}{|l}
$\widehat{\widehat{\varphi}}$ \\
$\dot{b}$ \\
0 \\
0 \\
$\dot{0}$ \\
$\dot{q}$
\end{tabular} & in & $\infty$ & $\begin{array}{r}\widehat{\widehat{N}} \\
\text { in } \\
\text { in }\end{array}$ & 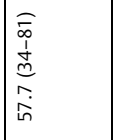 & 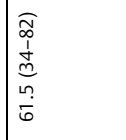 \\
\hline$z$ & - & - & - & n & o & m & in & i & m & n & - & - & $\stackrel{\sim}{2}$ & $\stackrel{0}{\circ}$ & $\supseteqq$ \\
\hline 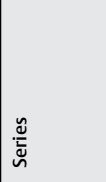 & 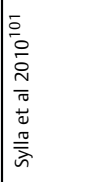 & 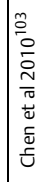 & 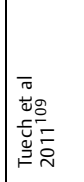 & 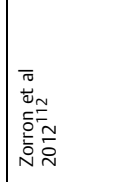 & 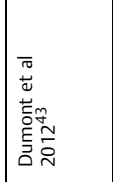 & 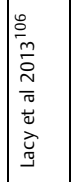 & 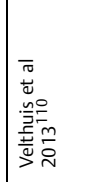 & 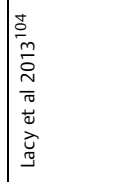 & 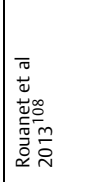 & 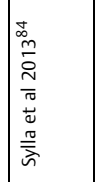 & 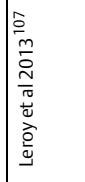 & 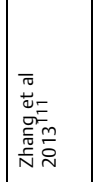 & 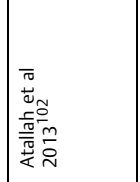 & 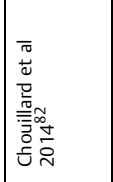 & \begin{tabular}{|l}
$\overline{\mathrm{v}}$ \\
$\overline{\mathrm{v}}$ \\
$\mathrm{u}$
\end{tabular} \\
\hline
\end{tabular}

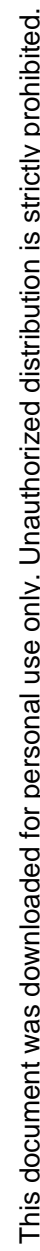



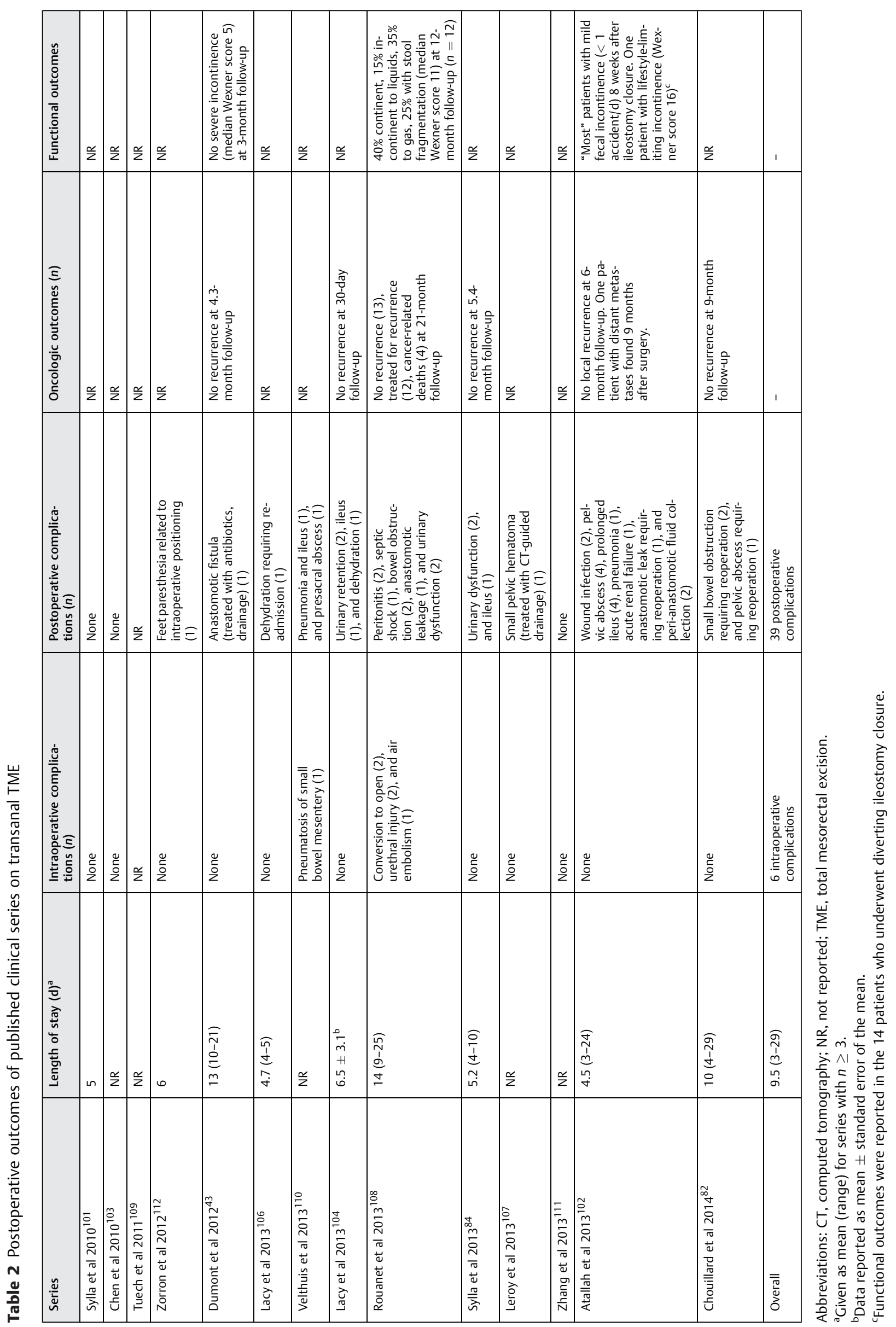
Oncologic results are similarly promising, with R0 resections achieved in $93.6 \%$ of the patients. Six patients had positive margins: four were high-risk patients with aggressive tumors reported by Rouanet et al and two had margins of $1 \mathrm{~mm}$ reported by Atallah et al. ${ }^{102,108}$ This rate of positive margins (5.5\%) is consistent with historical rates published in the literature for laparoscopic (1.2-16\%) and open (1.3-16\%) TME. ${ }^{1,36-38,40,42,113}$ Mean lymph node harvest was 17.9 nodes, which compares favorably to published rates of laparoscopic and open TME. ${ }^{1,37,40,42,113}$ Ninety-seven percent of the patients had complete $(n=101)$ or near-complete $(n=6)$ specimens, comparable to rates of oncologically satisfactory specimens after laparoscopic (72-88\%) and open (75-92\%) TME. ${ }^{1,37,42,113}$ Only two specimens were deemed incomplete; Atallah et al explained that these two specimens contained defects of $\geq 5 \mathrm{~mm}$ in the mesorectal envelopes but margins were negative and the patients did not recur during follow-up. ${ }^{102}$

Lacy et al, Dumont et al, Sylla et al, and Chouillard et al reported no tumor recurrence after follow-up periods of 30 days, 4.3 months, 5.4 months, and 9 months, respectively. ${ }^{43,82,84,104}$ Atallah et al found no local recurrence after median follow-up of 6 months; however, one patient developed distant metastases. ${ }^{102}$ Though that patient's final pathology was ypT3NO with complete TME specimen and negative margins and negative nodes, liver metastases were found 9 months after surgery and the patient required curative liver resection. In the high-risk patient cohort of Rouanet et al, 13 had no recurrence (43\%), 12 were treated for recurrence (40\%), and 4 died of cancer-related causes (13\%) during their 21-month follow-up period. ${ }^{108}$ Disease-free survival rates were $93.3 \%$ and $88.9 \%$ and overall survival rates were $96.6 \%$ and $80.5 \%$ at 12 - and 24 -month follow-up, respectively. Even in this patient population selected for high-risk tumors, these overall survival rates are similar to those after laparoscopic (85.8-90.7\% and $77.1-80.4 \%$ at 12 and 24 months, respectively) and open (80.5-92.7\% and 69.583.3\%) TME. $^{35,42}$

Preliminary results of functional outcomes after taTME demonstrate similar postoperative sphincter function when compared with laparoscopic or open TME. Rouanet et al, who performed partial ISR and used a rigid transanal platform, demonstrated a median Wexner score of 11 at 12-month follow-up, at which time $40 \%$ of the patients who underwent ileostomy closure were fully continent, $15 \%$ were incontinent to liquids, $35 \%$ were incontinent to gas, and $25 \%$ had stool fragmentation. ${ }^{108}$ This is comparable to published results of TME with ISR, which report median Wexner scores of 10.8 to 11. ${ }^{118-120}$ Dumont et al and Atallah et al both used flexible transanal platforms and externalized the specimen transabdominally; Dumont et al's cohort had a median Wexner score of 5 at 3 months after ileostomy reversal, while "most" of Atallah et al's cohort had mild fecal incontinence $(<1$ accident per day) at 8 weeks after ileostomy takedown. ${ }^{43,102}$ One patient reported by Atallah et al had lifestyle-limiting incontinence, with a Wexner score of 16 . These results are similar to functional outcomes after TME with low coloanal anastomoses (median Wexner score of 6.9). ${ }^{118}$
Thus, these clinical series demonstrate taTME to be feasible and oncologically safe. However, these preliminary results must be interpreted carefully, as they are based on aggregating data from a few studies with small cohorts, variable patient characteristics, different surgical techniques and equipment, and short follow-up periods. The morbidity, oncologic outcomes, and functional outcomes of transanal TME must be clarified by large trials with long-term follow-up before widespread adoption of transanal NOTES. Future trials may also elucidate any differences in postoperative pain, wound complications, and recovery time after NOTES versus conventional surgical approaches.

\section{Current Indications for Transanal Natural Orifice Transluminal Endoscopic Surgery Proctectomy and Total Mesorectal Excision}

Based on the current published experience with transanal NOTES proctectomy and TME, these complex procedures should be performed by surgeons with significant experience in laparoscopic or robotic TME, TEM or TAMIS, and ISR. ${ }^{121}$ Like with many minimally invasive techniques, a steep learning curve must be overcome to master transanal NOTES. In the largest cadaver series of transanal TME, Telem et al demonstrated decreased operative time $(p=0.13)$ and increased specimen length $(p=0.001)$ after the first five cadavers. ${ }^{97}$ This data suggests that procedural training with fresh human cadavers should be considered as the optimal training model for this approach. Alternatively, Buscaglia et al recently found that transanal NOTES sigmoidectomy training using an endoscopy-simulation model decreased operative time by $42 \%$ and may also be effective in gaining proficiency. ${ }^{122}$

Because of the limitations of currently available transanal instruments, NOTES colorectal procedures should be performed using a hybrid fashion, with transabdominal assistance. The same cadaver study by Telem et al compared three different approaches: transanal alone, transanal with transgastric assistance, and transanal with laparoscopic assistance. ${ }^{97}$ They found decreased operative time with the laparoscopic-assisted technique, as well as decreased complications, though the latter was not statistically significant. Laparoscopic assistance is helpful for mobilization of the splenic flexure, retraction of the colon during transanal rectal dissection, and identification of the ureters and pelvic nerves. While there have been 12 cases of unassisted transanal $\mathrm{TME},{ }^{107,111}$ platforms and instruments tailored to complex transanal surgery must be developed before pure transanal NOTES procedures can become commonplace.

The initial clinical experiences of transanal TME also demonstrate the importance of careful patient selection, particularly while many surgeons are early in the learning curve, and while larger studies on this technique continue to investigate long-term functional and oncologic outcomes of this approach. Currently, transanal NOTES procedures should only be performed in patients with benign disease or premalignant or resectable malignant tumors, preferably located in the low- or mid-rectum. ${ }^{17}$ These patients should have no history of extensive abdominal or pelvic surgery. 
Ideally, with increased surgeon expertise, taTME may be safely and consistently performed in patients likely to benefit most from a transanal approach. These are patients with characteristics that predict difficult transabdominal rectal dissections, including a narrow or deep pelvis, male gender, obesity, large prostate, low rectal tumor ( $<5 \mathrm{~cm}$ from $\mathrm{AV}$ ), and previous neoadjuvant radiation. ${ }^{102}$ Transanal TME may be particularly appropriate for these patients because of enhanced visualization and technically easier rectal and mesorectal dissection, which has the potential to improve the quality of the specimen and reduce the incidence of positive margins. ${ }^{123,124}$ Some groups, notably Rouanet et al and Dumont et al, have already begun investigating taTME in this subset of patients, with early results demonstrating equivalent morbidity and oncologic outcomes to previously published data from laparoscopic and open TME. ${ }^{43,108} \mathrm{Nev}-$ ertheless, taTME should not be performed on these high-risk patients until the surgical team has gained clinical expertise with NOTES techniques and until the long-term oncologic outcomes of these procedures are reported.

\section{Future Applications of Transanal Natural Orifice Transluminal Endoscopic Surgery}

Improvements in transanal platforms and specialized instruments have the potential to revolutionize applications of transanal NOTES. Pure, unassisted NOTES procedures will likely become easier to perform and may maximize the theoretical benefits of natural orifice surgery, namely decreased postoperative pain, wound complications, and recovery time. There have been 12 reported cases of pure transanal NOTES TME so far, 2 case reports and 10 patients who were part of a larger series. ${ }^{82,107,111}$ In all cases, the specimen was intact and $\geq 11$ nodes were collected. There were no intraoperative complications and three postoperative complications (a small pelvic hematoma treated with CT-guided drainage, a small bowel obstruction requiring reoperation, and a pelvic abscess requiring reoperation). Pure transanal NOTES has also been successfully reported for pull-through colectomy in six pediatric patients with long-segment intestinal aganglionosis, suggesting that pure NOTES may be used for indications beyond simply the removal of isolated lesions. ${ }^{125,126}$

Development of longer, more flexible scopes and instruments may also enable transanal NOTES procedures for more proximal colorectal lesions. Very few groups have pursued transanal colon resections because of the difficulty of dissection using currently available tools, though laparoscopicassisted transanal colectomy for benign disease has been shown to be safe and feasible by Lacy et al and Fuchs et al $^{59,99}$ In addition, Hall et al reported an excision of a rectal scar and successful transanal intraperitoneal creation of the colorectal anastomosis using a TEM platform, TEM instruments, and PDS suture, with no laparoscopic assistance. ${ }^{127}$ Though the resection margins were already closely approximated because of the small size of the scar and were thus under minimal tension, this case demonstrates the feasibility of suturing a colorectal anastomosis entirely intraperitoneally using a transanal platform, suggesting that more proximal transanal colorectal resections may be possible without the need to externalize the margins to perform a handsewn anastomosis. Nevertheless, the EURO-NOTES working group's 2012 recommendations state that transanal NOTES should currently only be utilized if the colotomy can be incorporated into the specimen or anastomosis, essentially restricting it to left-sided colon and rectal procedures. ${ }^{128}$

Another potential future application of NOTES is to biopsy sentinel lymph nodes (SLN) for rectal cancer. Currently, there is much debate over how to determine which patients with rectal cancer require radical resection and which will have similar oncologic outcomes after local excision. Radiologic staging has poor sensitivity for nodal spread, and local excision with transanal endoscopic surgery does not enable assessment of nodal status. $^{129}$ Transanal NOTES offers the possibility of obtaining more accurate staging by removing the tumor and then sampling SLN through the colotomy site, particularly appropriate given that $98 \%$ of the positive nodes are located in the laterodorsal mesorectum within $5 \mathrm{~cm}$ of the tumor. ${ }^{130,131}$ Transanal NOTES segmental sigmoid resection and SLN extraction was demonstrated to be feasible in a porcine model that used submucosal injection of methylene blue dye to locate the SLN. ${ }^{132}$ Arezzo reported a small human series $(n=3)$ in which indocyanine green was instilled in the submucosa around the rectal tumor, the tumor was removed, the fat dissected, and the SLN successfully removed, all via transanal NOTES. ${ }^{133}$ There were no complications specific to nodal dissection and removal, and the mesorectal fascia was preserved, maintaining oncologic integrity. Nevertheless, the utility of SLN biopsy in colorectal cancer is still under debate, as results are widely variable, with sensitivity rates ranging from 25 to $100 \%$ and false negative rates ranging from 0 to $75 \%{ }^{134}$

Another area of innovation with transanal NOTES is the incorporation of robotic technology. Robotics has been reported to improve visualization using 3-dimensional optical technology, enable ambidextrous movements, decrease tremor, and improve dexterity, particularly in confined spaces. ${ }^{135}$ Cadaver models have proven the feasibility of robotic TAMIS, ${ }^{136,137}$ and an initial clinical series $(n=16)$ by Hompes et al of robotic TAMIS for rectal lesions has demonstrated promising results. ${ }^{135}$ There has also been a cadaver series of laparoscopic-assisted robotic transanal TME, in which complete TME specimens were obtained in all four cadavers. ${ }^{138}$ Atallah et al reported the only clinical case thus far of robotic-assisted robotic transanal TME in a 51-year-old woman with a preoperatively staged T3N1 rectal tumor located $4 \mathrm{~cm}$ from the $A V .{ }^{83}$ The operative time was 381 minutes, there were no complications, negative margins were achieved, and the specimen quality was near-complete. These preliminary results demonstrate the safety and feasibility of robotic transanal NOTES; however, more trials are needed to elucidate the benefits and costs of adding robotic technology to the standard transanal endoscopic approach.

\section{Conclusion}

Transanal NOTES represents a paradigm shift in minimally invasive colorectal surgery, combining the benefits of TEM 
and TAMIS, ISR, and NOSE to minimize access trauma and optimize the quality and ease of rectal dissection. The transanal NOTES approach to TME for rectal cancer and proctocolectomy for benign disease has shown encouraging preliminary results; however, there is a need for larger trials to better characterize outcomes, advantages, disadvantages, and cost before widespread adoption. Currently, transanal NOTES procedures should only be performed in carefully selected patients with transabdominal assistance by surgeons experienced with laparoscopic TME, TEM or TAMIS, and ISR.

\section{References}

1 Kang SB, Park JW, Jeong SY, et al. Open versus laparoscopic surgery for mid or low rectal cancer after neoadjuvant chemoradiotherapy (COREAN trial): short-term outcomes of an openlabel randomised controlled trial. Lancet Oncol 2010;11(7): 637-645

2 Ohtani H, Tamamori Y, Azuma T, et al. A meta-analysis of the short- and long-term results of randomized controlled trials that compared laparoscopy-assisted and conventional open surgery for rectal cancer. J Gastrointest Surg 2011;15(8): 1375-1385

3 Fuchs KH, Meining A, von Renteln D, et al. Euro-NOTES Status Paper: from the concept to clinical practice. Surg Endosc 2013; 27(5):1456-1467

4 Mutter D, Dallemagne B, Perretta S, et al. Innovations in minimally invasive surgery: lessons learned from translational animal models. Langenbecks Arch Surg 2013;398(7):919-923

5 Telem DA, Berger DL, Bordeianou LG, Rattner DW, Sylla P. Update on transanal NOTES for rectal cancer: transitioning to human trials. Minim Invasive Surg 2012;2012:287613

6 Flora ED, Wilson TG, Martin IJ, O'Rourke NA, Maddern GJ. A review of natural orifice translumenal endoscopic surgery (NOTES) for intra-abdominal surgery: experimental models, techniques, and applicability to the clinical setting. Ann Surg 2008;247(4):583-602

7 Hochberger J, Lamadé W. Transgastric surgery in the abdomen: the dawn of a new era? Gastrointest Endosc 2005;62(2): 293-296

8 Lehmann KS, Ritz JP, Wibmer A, et al. The German registry for natural orifice translumenal endoscopic surgery: report of the first 551 patients. Ann Surg 2010;252(2):263-270

9 Salinas G, Saavedra L, Agurto H, et al. Early experience in human hybrid transgastric and transvaginal endoscopic cholecystectomy. Surg Endosc 2010;24(5):1092-1098

10 Zorron R, Palanivelu C, Galvão Neto MP, et al. International multicenter trial on clinical natural orifice surgery-NOTES IMTN study: preliminary results of 362 patients. Surg Innov 2010;17(2):142-158

11 Fong DG, Pai RD, Thompson CC. Transcolonic endoscopic abdominal exploration: a NOTES survival study in a porcine model. Gastrointest Endosc 2007;65(2):312-318

12 Fong DG, Ryou M, Pai RD, Tavakkolizadeh A, Rattner DW, Thompson CC. Transcolonic ventral wall hernia mesh fixation in a porcine model. Endoscopy 2007;39(10):865-869

13 Pai RD, Fong DG, Bundga ME, Odze RD, Rattner DW, Thompson CC. Transcolonic endoscopic cholecystectomy: a NOTES survival study in a porcine model (with video). Gastrointest Endosc 2006; 64(3):428-434

14 Ryou M, Fong DG, Pai RD, Tavakkolizadeh A, Rattner DW, Thompson CC. Dual-port distal pancreatectomy using a prototype endoscope and endoscopic stapler: a natural orifice transluminal endoscopic surgery (NOTES) survival study in a porcine model. Endoscopy 2007;39(10):881-887
15 Wilhelm D, Meining A, von Delius S, et al. An innovative, safe and sterile sigmoid access (ISSA) for NOTES. Endoscopy 2007;39(5): 401-406

16 Wagh MS, Thompson CC. Surgery insight: natural orifice transluminal endoscopic surgery-an analysis of work to date. Nat Clin Pract Gastroenterol Hepatol 2007;4(7):386-392

17 Sylla P. Current experience and future directions of completely NOTES colorectal resection. World J Gastrointest Surg 2010;2(6): 193-198

18 Merrifield BF, Wagh MS, Thompson CC. Peroral transgastric organ resection: a feasibility study in pigs. Gastrointest Endosc 2006; 63(4):693-697

19 Whiteford MH, Denk PM, Swanström LL. Feasibility of radical sigmoid colectomy performed as natural orifice translumenal endoscopic surgery (NOTES) using transanal endoscopic microsurgery. Surg Endosc 2007;21(10):1870-1874

20 Heald RJ. A new approach to rectal cancer. Br J Hosp Med 1979; 22(3):277-281

21 Arezzo A, Passera R, Scozzari G, Verra M, Morino M. Laparoscopy for rectal cancer reduces short-term mortality and morbidity: results of a systematic review and meta-analysis. Surg Endosc 2013;27(5):1485-1502

22 Asoglu O, Matlim T, Karanlik H, et al. Impact of laparoscopic surgery on bladder and sexual function after total mesorectal excision for rectal cancer. Surg Endosc 2009;23(2):296-303

23 Breukink S, Pierie J, Wiggers T. Laparoscopic versus open total mesorectal excision for rectal cancer. Cochrane Database Syst Rev 2006;18(4):CD005200

24 Dahlberg M, Glimelius B, Graf W, Påhlman L. Preoperative irradiation affects functional results after surgery for rectal cancer: results from a randomized study. Dis Colon Rectum 1998;41(5):543-549, discussion 549-551

25 Hida J, Yoshifuji T, Tokoro T, et al. Comparison of long-term functional results of colonic J-pouch and straight anastomosis after low anterior resection for rectal cancer: a five-year followup. Dis Colon Rectum 2004;47(10):1578-1585

26 Köhler A, Athanasiadis S, Ommer A, Psarakis E. Long-term results of low anterior resection with intersphincteric anastomosis in carcinoma of the lower one-third of the rectum: analysis of 31 patients. Dis Colon Rectum 2000;43(6):843-850

27 Machado M, Nygren J, Goldman S, Ljungqvist O. Similar outcome after colnic pouch and side-to-end anastomosis in low anterior resection for rectal cancer: a prospective randomized trial. Ann Surg 2003;238(2):214-220

28 Maggiori L, Panis Y. Is it time for a paradigm shift: "laparoscopy is now the best approach for rectal cancer"? Transl Gastrointest Cancer 2014;3(1):1-3

29 Schlachta CM, Mamazza J, Gregoire R, Burpee SE, Poulin EC. Could laparoscopic colon and rectal surgery become the standard of care? A review and experience with 750 procedures. Can J Surg 2003;46(6):432-440

30 Taylor GW, Jayne DG, Brown SR, et al. Adhesions and incisional hernias following laparoscopic versus open surgery for colorectal cancer in the CLASICC trial. Br J Surg 2010;97(1):70-78

31 Temple LK, Bacik J, Savatta SG, et al. The development of a validated instrument to evaluate bowel function after sphincter-preserving surgery for rectal cancer. Dis Colon Rectum 2005; 48(7):1353-1365

32 Vignali A, Fazio VW, Lavery IC, et al. Factors associated with the occurrence of leaks in stapled rectal anastomoses: a review of 1,014 patients. J Am Coll Surg 1997;185(2):105-113

33 Williamson ME, Lewis WG, Finan PJ, Miller AS, Holdsworth PJ, Johnston D. Recovery of physiologic and clinical function after low anterior resection of the rectum for carcinoma: myth or reality? Dis Colon Rectum 1995;38(4):411-418

34 Emhoff I, Lee G, Sylla P. Future directions in surgery for colorectal cancer: the evolving role of transanal endoscopic surgery. Colorectal Cancer 2014;3(2):195-213 
35 Green BL, Marshall HC, Collinson F, et al. Long-term follow-up of the Medical Research Council CLASICC trial of conventional versus laparoscopically assisted resection in colorectal cancer. Br J Surg 2013;100(1):75-82

36 Guillou PJ, Quirke P, Thorpe H, et al; MRC CLASICC trial group. Short-term endpoints of conventional versus laparoscopic-assisted surgery in patients with colorectal cancer (MRC CLASICC trial): multicentre, randomised controlled trial. Lancet 2005; 365(9472):1718-1726

37 van der Pas MH, Haglind E, Cuesta MA, et al; COlorectal cancer Laparoscopic or Open Resection II (COLOR II) Study Group. Laparoscopic versus open surgery for rectal cancer (COLOR II): short-term outcomes of a randomised, phase 3 trial. Lancet Oncol 2013;14(3):210-218

38 Aziz O, Constantinides V, Tekkis PP, et al. Laparoscopic versus open surgery for rectal cancer: a meta-analysis. Ann Surg Oncol 2006;13(3):413-424

39 Ito M, Sugito M, Kobayashi A, Nishizawa Y, Tsunoda Y, Saito N. Influence of learning curve on short-term results after laparoscopic resection for rectal cancer. Surg Endosc 2009;23(2): 403-408

40 Krane MK, Fichera A. Laparoscopic rectal cancer surgery: where do we stand? World J Gastroenterol 2012;18(46):6747-6755

41 Künzli BM, Friess H, Shrikhande SV. Is laparoscopic colorectal cancer surgery equal to open surgery? An evidence based perspective. World J Gastrointest Surg 2010;2(4):101-108

42 Lujan J, Valero G, Hernandez Q, Sanchez A, Frutos MD, Parrilla P. Randomized clinical trial comparing laparoscopic and open surgery in patients with rectal cancer. Br J Surg 2009;96(9): 982-989

43 Dumont F, Goéré D, Honoré C, Elias D. Transanal endoscopic total mesorectal excision combined with single-port laparoscopy. Dis Colon Rectum 2012;55(9):996-1001

$44 \mathrm{Kim}$ SJ, Ryu GO, Choi BJ, et al. The short-term outcomes of conventional and single-port laparoscopic surgery for colorectal cancer. Ann Surg 2011;254(6):933-940

45 Pedraza R, Aminian A, Nieto J, Faraj C, Pickron TB, Haas EM. Singleincision laparoscopic colectomy for cancer: short-term outcomes and comparative analysis. Minim Invasive Surg 2013; 2013:283438

46 Sirikurnpiboon S, Jivapaisarnpong P. Single-access laparoscopic rectal surgery is technically feasible. Minim Invasive Surg 2013; 2013:687134

47 Akmal Y, Baek JH, McKenzie S, Garcia-Aguilar J, Pigazzi A. Robotassisted total mesorectal excision: is there a learning curve? Surg Endosc 2012;26(9):2471-2476

48 Trastulli S, Farinella E, Cirocchi R, et al. Robotic resection compared with laparoscopic rectal resection for cancer: systematic review and meta-analysis of short-term outcome. Colorectal Dis 2012;14(4):e134-e156

49 Fung AK, Aly EH. Robotic colonic surgery: is it advisable to commence a new learning curve? Dis Colon Rectum 2013; 56(6):786-796

50 Awad ZT, Qureshi I, Seibel B, Sharma S, Dobbertien MA. Laparoscopic right hemicolectomy with transvaginal colon extraction using a laparoscopic posterior colpotomy: a 2-year series from a single institution. Surg Laparosc Endosc Percutan Tech 2011; 21(6):403-408

51 Choi GS, Park IJ, Kang BM, Lim KH, Jun SH. A novel approach of robotic-assisted anterior resection with transanal or transvaginal retrieval of the specimen for colorectal cancer. Surg Endosc 2009; 23(12):2831-2835

52 D'Hoore A, Wolthuis AM. Laparoscopic low anterior resection and transanal pull-through for low rectal cancer: a Natural Orifice Specimen Extraction (NOSE) technique. Colorectal Dis 2011;13 (Suppl 7):28-31

53 Franklin ME Jr, Liang S, Russek K. Natural orifice specimen extraction in laparoscopic colorectal surgery: transanal and transvaginal approaches. Tech Coloproctol 2013;17(Suppl 1): S63-S67

54 Lacy AM, Delgado S, Rojas OA, Almenara R, Blasi A, Llach J. MANOS radical sigmoidectomy: report of a transvaginal resection in the human. Surg Endosc 2008;22(7):1717-1723

55 Ooi BS, Quah HM, Fu CW, Eu KW. Laparoscopic high anterior resection with natural orifice specimen extraction (NOSE) for early rectal cancer. Tech Coloproctol 2009;13(1):61-64

56 Torres RA, Orban RD, Tocaimaza L, Vallejos Pereira G, Arévalo JR. Transvaginal specimen extraction after laparoscopic colectomy. World J Surg 2012;36(7):1699-1702

57 Wang Q Wang C, Sun DH, Kharbuja P, Cao XY. Laparoscopic total mesorectal excision with natural orifice specimen extraction. World J Gastroenterol 2013;19(5):750-754

58 Arezzo A, Zornig C, Mofid $\mathrm{H}$, et al. The EURO-NOTES clinical registry for natural orifice transluminal endoscopic surgery: a 2year activity report. Surg Endosc 2013;27(9):3073-3084

59 Fuchs K-H, Breithaupt W, Varga G, Schulz T, Reinisch A, Josipovic N. Transanal hybrid colon resection: from laparoscopy to NOTES. Surg Endosc 2013;27(3):746-752

60 Park JS, Choi GS, Kim HJ, Park SY, Jun SH. Natural orifice specimen extraction versus conventional laparoscopically assisted right hemicolectomy. Br J Surg 2011;98(5):710-715

61 Leung AL, Cheung HY, Fok BK, Chung CC, Li MK, Tang CN. Prospective randomized trial of hybrid NOTES colectomy versus conventional laparoscopic colectomy for left-sided colonic tumors. World J Surg 2013;37(11):2678-2682

62 Franklin ME Jr, Liang S, Russek K. Integration of transanal specimen extraction into laparoscopic anterior resection with total mesorectal excision for rectal cancer: a consecutive series of 179 patients. Surg Endosc 2013;27(1):127-132

63 Liang S, Franklin M Jr. Transanal versus Transabdominal Specimen Extraction with Laparoscopic Low Anterior Resection: A Comparative Analysis on 432 Patients with Rectal Cancer. Baltimore, MD: Society of American Gastrointestinal and Endoscopic Surgeons (SAGES) 2013; 2013

64 Buess G, Theiss R, Günther M, Hutterer F, Pichlmaier H. Endoscopic surgery in the rectum. Endoscopy 1985;17(1):31-35

65 Buess G, Theiss R, Hutterer F, et al. Transanal endoscopic surgery of the rectum - testing a new method in animal experiments [in German]. Leber Magen Darm 1983;13(2):73-77

66 Moore JS, Cataldo PA, Osler T, Hyman NH. Transanal endoscopic microsurgery is more effective than traditional transanal excision for resection of rectal masses. Dis Colon Rectum 2008;51(7): 1026-1030, discussion 1030-1031

67 Langer C, Liersch T, Süss M, et al. Surgical cure for early rectal carcinoma and large adenoma: transanal endoscopic microsurgery (using ultrasound or electrosurgery) compared to conventional local and radical resection. Int J Colorectal Dis 2003;18(3): 222-229

68 Winde G, Nottberg H, Keller R, Schmid KW, Bünte H. Surgical cure for early rectal carcinomas (T1). Transanal endoscopic microsurgery vs. anterior resection. Dis Colon Rectum 1996;39(9): 969-976

69 Albert MR, Atallah SB, deBeche-Adams TC, Izfar S, Larach SW. Transanal minimally invasive surgery (TAMIS) for local excision of benign neoplasms and early-stage rectal cancer: efficacy and outcomes in the first 50 patients. Dis Colon Rectum 2013;56(3): 301-307

70 Alves FE, Costa P, Guerra J. Transanal minimally invasive surgery with single-port (TAMIS) for the management of rectal neoplasms: a pilot study. J Coloproctol 2012;32(4):402-406

71 Atallah SB, Albert MR. Transanal minimally invasive surgery (TAMIS) versus transanal endoscopic microsurgery (TEM): is one better than the other? Surg Endosc 2013;27(12):4750-4751

72 Rega D, Cardone E, Montesarchio L, et al. Transanal minimally invasive surgery with single-port laparoscopy for rectal tumors. Eur J Surg Oncol 2012;38(10):982 
73 National Comprehensive Cancer Network. Clinical Practice Guidelines in Oncology. Rectal Cancer. Fort Washington, PA: National Comprehensive Cancer Network; 2013

74 Bach SP, Hill J, Monson JR, et al; Association of Coloproctology of Great Britain and Ireland Transanal Endoscopic Microsurgery (TEM) Collaboration. A predictive model for local recurrence after transanal endoscopic microsurgery for rectal cancer. Br J Surg 2009;96(3):280-290

75 Bentrem DJ, Okabe S, Wong WD, et al. T1 adenocarcinoma of the rectum: transanal excision or radical surgery? Ann Surg 2005; 242(4):472-477, discussion 477-479

76 Greenberg JA, Shibata D, Herndon JE II, Steele GD Jr, Mayer R, Bleday R. Local excision of distal rectal cancer: an update of cancer and leukemia group B 8984. Dis Colon Rectum 2008;51(8): 1185-1191, discussion 1191-1194

77 Monson JR, Weiser MR, Buie WD, et al; Standards Practice Task Force of the American Society of Colon and Rectal Surgeons. Practice parameters for the management of rectal cancer (revised). Dis Colon Rectum 2013;56(5):535-550

78 Nash GM, Weiser MR, Guillem JG, et al. Long-term survival after transanal excision of T1 rectal cancer. Dis Colon Rectum 2009; 52(4):577-582

79 Nesbakken A, Nygaard K, Westerheim O, Mala T, Lunde OC. Local recurrence after mesorectal excision for rectal cancer. Eur J Surg Oncol 2002;28(2):126-134

80 Peng J, Chen W, Sheng W, et al. Oncological outcome of T1 rectal cancer undergoing standard resection and local excision. Colorectal Dis 2011;13(2):e14-e19

81 You YN, Baxter NN, Stewart A, Nelson H. Is the increasing rate of local excision for stage I rectal cancer in the United States justified?: a nationwide cohort study from the National Cancer Database Ann Surg 2007;245(5):726-733

82 Chouillard E, Chahine E, Khoury G, et al. NOTES total mesorectal excision (TME) for patients with rectal neoplasia: a preliminary experience. Surg Endosc 2014;28(11):3150-3157 Epub ahead of print

83 Atallah S, Nassif G, Polavarapu H, et al. Robotic-assisted transanal surgery for total mesorectal excision (RATS-TME): a description of a novel surgical approach with video demonstration. Tech Coloproctol 2013;17(4):441-447

84 Sylla P, Bordeianou LG, Berger D, et al. A pilot study of natural orifice transanal endoscopic total mesorectal excision with laparoscopic assistance for rectal cancer. Surg Endosc 2013;27(9): 3396-3405

85 Schiessel R, Karner-Hanusch J, Herbst F, Teleky B, Wunderlich M. Intersphincteric resection for low rectal tumours. Br J Surg 1994; 81(9):1376-1378

86 Akagi Y, Shirouzu K, Ogata Y, Kinugasa T. Oncologic outcomes of intersphincteric resection without preoperative chemoradiotherapy for very low rectal cancer. Surg Oncol 2013;22(2):144-149

87 Marks G, Mohiuddin M, Goldstein SD. Sphincter preservation for cancer of the distal rectum using high dose preoperative radiation. Int J Radiat Oncol Biol Phys 1988;15(5):1065-1068

88 Liyanage C, Ramwell A, Harris GJ, Levy BF, Simson JN. Transanal endoscopic microsurgery: a new technique for completion proctectomy. Colorectal Dis 2013;15(9):e542-e547

89 Bhattacharjee HK, Buess GF, Becerra Garcia FC, et al. A novel single-port technique for transanal rectosigmoid resection and colorectal anastomosis on an ex vivo experimental model. Surg Endosc 2011;25(6):1844-1857

90 Bhattacharjee HK, Kirschniak A, Storz P, Wilhelm P, Kunert W. Transanal endoscopic microsurgery-based transanal access for colorectal surgery: experience on human cadavers. J Laparoendosc Adv Surg Tech A 2011;21(9):835-840

91 Fajardo AD, Hunt SR, Fleshman JW, Mutch MG. Video. Transanal single-port low anterior resection in a cadaver model. Surg Endosc 2010;24(7):1765
92 Leroy J, Cahill RA, Perretta S, Forgione A, Dallemagne B, Marescaux J. Natural orifice translumenal endoscopic surgery (NOTES) applied totally to sigmoidectomy: an original technique with survival in a porcine model. Surg Endosc 2009;23(1):24-30

93 Rieder E, Spaun GO, Khajanchee YS, et al. A natural orifice transrectal approach for oncologic resection of the rectosigmoid: an experimental study and comparison with conventional laparoscopy. Surg Endosc 2011;25(10):3357-3363

94 Sohn DK, Jeong SY, Park JW, et al. Comparative study of NOTES rectosigmoidectomy in a swine model: E-NOTES vs. P-NOTES. Endoscopy 2011;43(6):526-532

95 Sylla P, Sohn DK, Cizginer S, et al. Survival study of natural orifice translumenal endoscopic surgery for rectosigmoid resection using transanal endoscopic microsurgery with or without transgastric endoscopic assistance in a swine model. Surg Endosc 2010;24(8):2022-2030

96 Sylla P, Willingham FF, Sohn DK, Gee D, Brugge WR, Rattner DW. NOTES rectosigmoid resection using transanal endoscopic microsurgery (TEM) with transgastric endoscopic assistance: a pilot study in swine. J Gastrointest Surg 2008;12(10):1717-1723

97 Telem DA, Han KS, Kim M-C, et al. Transanal rectosigmoid resection via natural orifice translumenal endoscopic surgery (NOTES) with total mesorectal excision in a large human cadaver series. Surg Endosc 2013;27(1):74-80

98 Trunzo JA, Delaney CP. Natural orifice proctectomy using a transanal endoscopic microsurgical technique in a porcine model. Surg Innov 2010;17(1):48-52

99 Lacy AM, Saavedra-Perez D, Bravo R, Adelsdorfer C, Aceituno M, Balust J. Minilaparoscopy-assisted natural orifice total colectomy: technical report of a minilaparoscopy-assisted transrectal resection. Surg Endosc 2012;26(7):2080-2085

100 Wolthuis AM, de Buck van Overstraeten A, D'Hoore A. Dynamic article: transanal rectal excision: a pilot study. Dis Colon Rectum 2014;57(1):105-109

101 Sylla P, Rattner DW, Delgado S, Lacy AM. NOTES transanal rectal cancer resection using transanal endoscopic microsurgery and laparoscopic assistance. Surg Endosc 2010;24(5):1205-1210

102 Atallah S, Martin-Perez B, Albert M, et al. Transanal minimally invasive surgery for total mesorectal excision (TAMIS-TME): results and experience with the first 20 patients undergoing curative-intent rectal cancer surgery at a single institution. Tech Coloproctol 2013; Epub ahead of print

103 Chen Y, Hu M, Lei J, Chen J, Li J. NOTES transanal endoscopic total mesorectal excision for rectal cancer. China Journal of Endoscopy 2010;16(12):1261-1265

104 de Lacy AM, Rattner DW, Adelsdorfer C, et al. Transanal natural orifice transluminal endoscopic surgery (NOTES) rectal resection: "down-to-up" total mesorectal excision (TME)-short-term outcomes in the first 20 cases. Surg Endosc 2013;27(9): 3165-3172

105 Emhoff IA, Lee GC, Sylla P. Transanal colorectal resection using natural orifice translumenal endoscopic surgery (NOTES). Dig Endosc 2014;26(Suppl 1):29-42

106 Lacy AM, Adelsdorfer C, Delgado S, Sylla P, Rattner DW. Minilaparoscopy-assisted transrectal low anterior resection (LAR): a preliminary study. Surg Endosc 2013;27(1):339-346

107 Leroy J, Barry BD, Melani A, Mutter D, Marescaux J. No-scar transanal total mesorectal excision: the last step to pure NOTES for colorectal surgery. JAMA Surg 2013;148(3):226-230, discussion 231

108 Rouanet P, Mourregot A, Azar CC, et al. Transanal endoscopic proctectomy: an innovative procedure for difficult resection of rectal tumors in men with narrow pelvis. Dis Colon Rectum 2013; 56(4):408-415

109 Tuech JJ, Bridoux V, Kianifard B, et al. Natural orifice total mesorectal excision using transanal port and laparoscopic assistance. Eur J Surg Oncol 2011;37(4):334-335 
110 Velthuis S, van den Boezem PB, van der Peet DL, Cuesta MA, Sietses C. Feasibility study of transanal total mesorectal excision. Br J Surg 2013;100(6):828-831, discussion 831

111 Zhang H, Zhang YS, Jin XW, Li MZ, Fan JS, Yang ZH. Transanal single-port laparoscopic total mesorectal excision in the treatment of rectal cancer. Tech Coloproctol 2013;17(1):117-123

112 Zorron R, Phillips HN, Coelho D, Flach L, Lemos FB, Vassallo RC. Perirectal NOTES access: "down-to-up" total mesorectal excision for rectal cancer. Surg Innov 2012;19(1):11-19

113 Lujan J, Valero G, Biondo S, Espin E, Parrilla P, Ortiz H. Laparoscopic versus open surgery for rectal cancer: results of a prospective multicentre analysis of 4,970 patients. Surg Endosc 2013;27(1): 295-302

114 Targarona EM, Balague C, Pernas JC, et al. Can we predict immediate outcome after laparoscopic rectal surgery? Multivariate analysis of clinical, anatomic, and pathologic features after 3dimensional reconstruction of the pelvic anatomy. Ann Surg 2008;247(4):642-649

115 Leroy J, Jamali F, Forbes L, et al. Laparoscopic total mesorectal excision (TME) for rectal cancer surgery: long-term outcomes. Surg Endosc 2004;18(2):281-289

116 Kim NK, Aahn TW, Park JK, et al. Assessment of sexual and voiding function after total mesorectal excision with pelvic autonomic nerve preservation in males with rectal cancer. Dis Colon Rectum 2002;45(9):1178-1185

117 Maurer CA. Urinary and sexual function after total mesorectal excision. Recent Results Cancer Res 2005;165:196-204

118 Bretagnol F, Rullier E, Laurent C, Zerbib F, Gontier R, Saric J. Comparison of functional results and quality of life between intersphincteric resection and conventional coloanal anastomosis for low rectal cancer. Dis Colon Rectum 2004;47(6):832-838

119 Dumont F, Ayadi M, Goéré D, Honoré C, Elias D. Comparison of fecal continence and quality of life between intersphincteric resection and abdominoperineal resection plus perineal colostomy for ultra-low rectal cancer. J Surg Oncol 2013;108(4):225-229

120 Rouanet P, Saint-Aubert B, Lemanski C, et al. Restorative and nonrestorative surgery for low rectal cancer after high-dose radiation: long-term oncologic and functional results. Dis Colon Rectum 2002;45(3):305-313, discussion 313-315

121 Lacy AM, Adelsdorfer C. Totally transrectal endoscopic total mesorectal excision (TME). Colorectal Dis 2011;13 (Suppl 7):43-46

122 Buscaglia JM, Karas J, Palladino N, et al. Simulated transanal NOTES sigmoidectomy training improves the responsiveness of surgical endoscopists. Gastrointest Endosc 2014;80(1):126-132 Epub ahead of print

123 Nahar Al-Furaji HH, Sehgal R, Mansoor T, Burke J, Cahill RA. Transanal and transrectal operations for excisional surgery of the low and mid rectum (with video). Surg Technol Int 2014; 24:124-132

124 Sehgal R, Cahill RA. Advanced laparoscopic surgery for colorectal disease: NOTES/NOSE or single port? Best Pract Res Clin Gastroenterol 2014;28(1):81-96
125 Li N, Zhang W, Yu D, et al. NOTES for surgical treatment of longsegment Hirschsprung's disease: report of three cases. J Laparoendosc Adv Surg Tech A 2013;23(12):1020-1023

126 Vahdad MR, Foroutan A, Najafi SM, et al. Totally transanal LESS pull-through colectomy: a novel approach for avoiding abdominal wall incision in children with long-segment intestinal aganglionosis. J Laparoendosc Adv Surg Tech A 2013;23(3): 276-280

127 Hall DJ, Farmer KC, Roth HS, Warrier SK. Transanal endoscopic microsurgery colorectal anastomosis: a critical step to natural orifice colorectal surgery in humans. Dis Colon Rectum 2014; 57(4):549-552

128 Meining A, Spaun G, Fernández-Esparrach G, et al. NOTES in Europe: summary of the working group reports of the 2012 EURO-NOTES meeting. Endoscopy 2013;45(3):214-217

$129 \mathrm{Kim}$ H, Lim JS, Choi JY, et al. Rectal cancer: comparison of accuracy of local-regional staging with two- and three-dimensional preoperative 3-T MR imaging. Radiology 2010;254(2):485-492

130 Engelen SM, Beets-Tan RG, Lahaye MJ, Kessels AG, Beets GL. Location of involved mesorectal and extramesorectal lymph nodes in patients with primary rectal cancer: preoperative assessment with MR imaging. Eur J Surg Oncol 2008;34(7): 776-781

131 Koh DM, Brown G, Temple L, et al. Distribution of mesorectal lymph nodes in rectal cancer: in vivo MR imaging compared with histopathological examination. Initial observations. Eur Radiol 2005;15(8):1650-1657

132 Cahill RA, Perretta S, Forgione A, Leroy J, Dallemagne B, Marescaux J. Multimedia article. Combined sentinel node biopsy and localized sigmoid resection entirely by natural orifice transluminal endoscopic surgery: a new challenge to the old paradigm. Dis Colon Rectum 2009;52(4):725

133 Arezzo A. Fluorescence-guided mesorectal lymph node sampling during transanal endoscopic microsurgery for early rectal tumours. Amsterdam: United European Gastroenterology Week; 2012

134 Cahill RA, Leroy J, Marescaux J. Could lymphatic mapping and sentinel node biopsy provide oncological providence for local resectional techniques for colon cancer? A review of the literature. BMC Surg 2008;8:17

135 Hompes R, Rauh SM, Ris F, Tuynman JB, Mortensen NJ. Robotic transanal minimally invasive surgery for local excision of rectal neoplasms. Br J Surg 2014;101(5):578-581

136 Atallah SB, Albert MR, deBeche-Adams TH, Larach SW. Robotic TransAnal Minimally Invasive Surgery in a cadaveric model. Tech Coloproctol 2011;15(4):461-464

137 Hompes R, Rauh SM, Hagen ME, Mortensen NJ. Preclinical cadaveric study of transanal endoscopic da Vinci ${ }^{\circledR}$ surgery. $\mathrm{Br} \mathrm{J}$ Surg 2012;99(8):1144-1148

138 Gomez Ruiz M, Martin Parra I, Calleja Iglesias A, et al. Preclinical cadaveric study of transanal robotic proctectomy with total mesorectal excision combined with laparoscopic assistance. Int J Med Robot 2014; Epub ahead of print 